\title{
SATISFAÇÃO COM OS CUIDADOS E FELICIDADE EM IDOSOS INSTITUCIONALIZADOS
}

\author{
Ana Galvão \\ Instituto Politécnico de Bragança \\ UICISA: E \\ anagalvao@ipb.pt \\ André Silva \\ Instituto Politécnico de Bragança \\ Maria José Gomes \\ Instituto Politécnico de Bragança \\ UICISA: E \\ mgomes@ipb.pt \\ Marco Pinheiro \\ ISCTE - Instituto Universitário de Lisboa \\ marco.paulo.pinheiro@iscte-iul.pt
}

Recepción Artículo: 5 noviembre 2020

Admisión Evaluación: 5 noviembre 2020

Informe Evaluador 1: 12 noviembre 2020

Informe Evaluador 2: 17 noviembre 2020

Aprobación Publicación: 27 noviembre 2020

\section{RESUMO}

A satisfação e a felicidade são aspetos determinantes no processo de envelhecimento saudável e gratificante. 0 recurso à institucionalização dos mais velhos é uma realidade cada vez mais frequente nas sociedades desenvolvidas, acarretando diversas consequências na vida do idoso. Perante esta transiç̧ão de contextos vivenciada pelo idoso, torna-se oportuno avaliar a satisfação perante os cuidados prestados e o nível de felicidade, assim, objetivou-se analisar a satisfação com os cuidados e a felicidade de idosos institucionalizados. Realizou-se um estudo de caráter exploratório, descritivo e inferencial, numa amostra constituída por 61 idosos institucionalizados em quatro Estruturas Residenciais para Pessoas Idosas, do concelho de Santa Marta de Penaguião. Aplicouse um inquérito por questionário com o objetivo de recolher dados de contexto Sociodemográfico, Situacional, Familiar e Clínicos. Para avaliar o nível de Satisfação com os cuidados e Felicidade foram utilizadas as seguintes escalas: Escala da Felicidade de Oxford e a Escala de Avaliação da Satisfação em Estrutura Residencial para Pessoas Idosas desenvolvido pelo Instituto da Segurança Social. Concluiu-se que a maioria da amostra é do sexo feminino (75.4\%), a faixa etária predominante situa-se entre os 85 e 89 anos (29.5\%) são provenientes de aldeia (77\%) e viúvos (65.6\%) sem nunca terem frequentado a escola (54.1\%). Relativamente ao tipo de reforma, os idosos recebem pensões por limite de idade e pensão do cônjuge (31.1\%) e pensão do cônjuge e invalidez (31.1\%) sendo que a maioria (62.3\%) recebe mensalmente um valor entre 251 a 500 euros e encontram-se satis- 


\section{SATISFAÇÃO COM OS CUIDADOS E FELICIDADE EM IDOSOS INSTITUCIONALIZADOS}

feitos com esse valor (57.4\%). De modo geral, os resultados obtidos através das escalas utilizadas permitem concluir que os participantes, maioritariamente, encontram-se satisfeitos e felizes com a sua vivência de institucionalização. 0 envelhecimento da população é, antes de tudo, uma estória de sucesso que decorre dos avanços médico, tecnológico e social.

Palavras chave: felicidade; institucionalização; idoso; envelhecimento saudável; satisfação pessoal

\section{ABSTRACT}

Satisfaction with the provided care and happiness of institutionalised senior citizens. Satisfaction and happiness are determining aspects in the healthy and rewarding aging process. The recourse to the institutionalisation of the elderly is an increasingly frequent reality in developed societies, with several consequences in the life of the elderly. In view of this transition of contexts experienced by the elderly, it is opportune to assess satisfaction with the care provided and the level of happiness, thus, the objective was to analyse the satisfaction with the care and happiness of institutionalized elderly people. An exploratory, descriptive and inferential study was carried out, in a sample consisting of 61 institutionalized elderly people in four Residential Structures for Elderly People, in the municipality of Santa Marta de Penaguião. A questionnaire survey was applied in order to collect data from the Sociodemographic, Situational, Family and Clinical context. To assess the level of Satisfaction with care and Happiness, the following scales were used: Oxford Happiness Scale and the Residential Structure Satisfaction Assessment Scale for Elderly People developed by the Social Security Institute. It was concluded that the majority of the sample is female $(75.4 \%)$, the predominant age group is between 85 and 89 years old $(29.5 \%)$ coming from the village (77\%) and widowers (65.6\%) without ever attended school (54.1\%). Regarding the type of retirement, the elderly receive pensions for age limit and spouse's pension (31.1\%) and spouse's pension and disability (31.1\%), with the majority (62.3\%) receiving monthly between 251 and 500 euros and are satisfied with this value (57.4\%). In general, the results obtained through the scales allow us to conclude that the participants, mostly, are satisfied and happy with their experience of institutionalisation. The aging of the population is, above all, a success story that stems from medical, technological and social advances.

Keywords: happiness; institutionalisation; senior citizens; healthy aging; personal satisfaction

\section{ENQUADRAMENTO TEÓRICO}

Vivemos numa população cada vez mais envelhecida, é um facto incontornável e transversal. 0 envelhecimento da população é uma das maiores conquistas da humanidade e simultaneamente um dos maiores desafios da sociedade. 0 envelhecimento demográfico atualmente é assumido como um tema de máxima importância a nível mundial e consequentemente emerge a necessidade de mais respostas sociais para os idosos, incluindo a institucionalização.

Envelhecer numa instituição torna-se cada vez mais uma realidade. A institucionalização tem vindo a tornarse num dos sinais mais vincados do envelhecimento, tornando-se cada vez mais frequente este facto que os idosos vivenciam por motivos diversos, a maioria das vezes, não conseguem encontrar no núcleo familiar uma resposta favorável às suas necessidades básicas. A institucionalização representa para a pessoa idosa uma mudança significativa nos seus contextos de vida, nos quais participou ativamente e agora vê-se obrigada a desvincularse deles. 0 idoso encontra-se perante uma realidade completamente nova e, por vezes, assustadora, com a qual nem sempre consegue estabelecer uma relação equilibrada e tranquila, havendo, no entanto, alguns estudos que concluem que a institucionalização contribui para um aumento da felicidade dos idosos (Kok et al., 2015; K. Moore et al., 2007; Rinnan et al., 2018).

0 paradigma lifespan, proposto por Paul Baltes, em 1987, descreve o processo de desenvolvimento e os princípios gerais do processo de envelhecimento. 0 desenvolvimento humano é encarado como um processo contínuo, multidimensional e multidirecional de transformações influenciadas por fatores genético-biológicos e socioculturais, de natureza normativa e não normativa (Baltes \& Baltes, 2010). 
Este paradigma integra uma sucessão de modificações previsíveis e não previsíveis, de natureza genéticobiológica e psicossocial. Denomina transformações não normativas, aquelas que são desencadeadas por influências não previsíveis, que são provocadas pela influência de agentes biológicos e sociais, como por exemplo a perda do emprego, a viuvez na idade adulta, acidentes e doenças.

Como exemplos das transformações normativas, podemos considerar a maturação neurológica dos cinco primeiros anos, a puberdade, o climatério e a menopausa, que ocorrem ao longo do ciclo vital e também as alterações associadas aos processos de socialização ao qual os indivíduos estão sujeitos, como o caso da entrada para escola, o casamento e a aposentação, respetivamente.

No que concerne ao processo do envelhecimento, este processo é um acontecimento multidimensional, complexo, dinâmico, que não pode ser evitado. 0 mesmo ocorre de forma natural, progressiva, previsível e irreversível, sendo comum a todos, provocando não só mudanças ao longo da vida, envolvendo também diferenciação, crescimento e desenvolvimento do indivíduo.

As evidências corroboram que nesta perspetiva multidimensional, o processo de envelhecimento decorre da correlação de múltiplos processos de desenvolvimento, sofrendo influência de fatores: biológicos, sociais e psicológicos, que interagem entre si de modo constante.

Se optarmos pela ideia do processo de envelhecimento como uma experiência positiva, uma vida mais longa, estamos perante o conceito de Envelhecimento ativo, proposto pela Organização Mundial da Saúde, na década de 90, tendo adotado o termo, envelhecimento ativo, para identificar o envelhecimento como um processo de otimização das oportunidades de saúde, participação e segurança, com o objetivo de melhorar a qualidade de vida à medida que as pessoas envelhecem.

Analisando a estratégia nacional para o envelhecimento ativo e saudável 2017-2025, surge-nos mencionado como atributo do processo de envelhecimento ser um processo ativo (Direção-Geral da Saúde, 2017). Referindose este atributo, à participação contínua das pessoas mais velhas, nas questões sociais, económicas, culturais, espirituais e civis, e não somente à capacidade de estar fisicamente ativo ou de fazer parte da força de trabalho. As pessoas mais velhas que se aposentam e aquelas que apresentam alguma doença ou vivem com alguma necessidade especial podem continuar a contribuir ativamente para seus familiares, companheiros, comunidades e países. 0 objetivo do envelhecimento ativo é aumentar a expetativa de uma vida saudável, do bem estar geral, e a qualidade de vida para todas as pessoas que estão a envelhecer, inclusive as que são frágeis, fisicamente incapacitadas e que requerem cuidados (WHO, 2002).

A abordagem do processo de envelhecimento ativo e gratificante, baseia-se no reconhecimento dos direitos humanos das pessoas mais velhas e nos princípios de independência, participação, dignidade, assistência e autorrealização estabelecidos pela Organização das Nações Unidas.

Em 2015, o relatório mundial, sobre o Envelhecimento Saudável define este conceito, como o processo de desenvolvimento e manutenção da capacidade funcional que permite 0 bem-estar em idade avançada (WHO, 2015).

Na proposta atrás referida sobre a estratégia nacional para o envelhecimento ativo e saudável 2017-2025, a Direção-Geral da Saúde, conceptualiza o envelhecimento saudável como o processo de desenvolvimento e manutenção da capacidade funcional, que contribui para o bem-estar das pessoas idosas, sendo a capacidade funcional o resultado da interação das capacidades intrínsecas da pessoa (físicas e mentais) com o meio (Direção-Geral da Saúde, 2017). Sendo o objetivo principal o bem-estar, um conceito holístico que contempla todos os elementos e componentes da vida valorizados pela pessoa. Assim, mais do que o resultado do sucesso e da motivação individual, o envelhecimento saudável é o reflexo dos hábitos de vida, do suporte e das oportunidades garantidas pela sociedade para a manutenção da funcionalidade das pessoas idosas e para permitir que vivenciem aquilo que valorizam (WHO, 2015).

0 processo do envelhecimento saudável e gratificante, depende de uma diversidade de fatores determinantes que envolvem os indivíduos, as famílias e as comunidades. Estes fatores podem ser agrupados em: fatores eco- 


\section{SATISFAÇÃO COM OS CUIDADOS E FELICIDADE EM IDOSOS INSTITUCIONALIZADOS}

nómicos (são identificados três aspetos dos determinantes económicos que têm um efeito particularmente relevante sobre o processo do envelhecimento ativo, 0 salário, 0 trabalho, e a proteção social); contexto ambiental (condições de higiene e segurança no ambiente físico, prevenção de quedas); fatores sociais (rede de apoio social, oportunidades de educação e aprendizagem permanente, proteção contra a violência e maus-tratos, prevenção da solidão e isolamento social); serviços de saúde e sociais (os sistemas de saúde necessitam ter uma perspetiva sólida do trajeto do ciclo de vida que vise a promoção da saúde, prevenção de doenças e acesso equitativo aos cuidados primários e integrados, coordenados de forma eficaz. Um enfoque marcado para os serviços de saúde mental, que desempenham um papel crucial no processo do envelhecimento saudável, devem ser uma parte integral na assistência a longo prazo. Deve dar-se uma atenção especial aos subdiagnósticos de doença mental (especialmente depressão) e às taxas de suicídio em idosos; fatores pessoais (aspetos ligados à biologia e à genética; fatores psicológicos e mentais); estilos de vida e comportamentos de saúde (a adoção de estilos de vida saudáveis e a participação ativa nos autocuidados para a saúde são importantes em todos os estádios do ciclo vital, o envolvimento em atividades físicas adequadas, alimentação saudável, a abstinência do fumo e do álcool, 0 uso seguro e racional do medicamento, podem prevenir doenças e o declínio funcional, aumentar a longevidade e a qualidade de vida do indivíduo).

Consideramos como determinante transversal ao processo do envelhecimento, o macrossistema identificado por Bronfenbrenner o qual engloba os aspetos culturais e económicos dum país. A cultura é um fator determinante transversal para compreendermos o processo de envelhecimento ativo, pois, modela a nossa forma de envelhecer, influenciando todos os outros fatores determinantes do processo do envelhecimento. Os valores culturais e as tradições determinam fortemente como uma sociedade encara as pessoas mais velhas e 0 processo de envelhecimento (Bronfenbrenner, 1977, 1986, 2005; Bronfenbrenner \& Ceci, 1994; Bronfenbrenner \& Morris, 2007).

Outro determinante transversal ao processo do envelhecimento e gratificante ativo poderá ser 0 género. Constatamos um elevado número de cuidadores informais pertencentes ao género feminino.

0 envelhecimento saudável é um processo contínuo e constitui um desafio que a sociedade não pode ignorar. Os idosos são podem ser considerados como um grupo homogéneo, pois na realidade, o processo de enveIhecer encerra em si uma heterogeneidade. Esta afirmação encontra suporte nas afirmações da OMS, mencionado que que as incapacidades que vão surgindo com o envelhecimento não estão diretamente relacionadas com esse envelhecimento. E que estas incapacidades decorrem muitas vezes de situações que aparecem ao longo da vida e que não raro são modificáveis por nós (ou seja através da prática de comportamentos de saúde e de estilos de vida).

Segundo Neri (2006) a pessoa idosa tem uma história vivida e um modo de compreender o mundo e a si mesma; os seus valores, metas, motivações e expetativas e um conjunto de repertórios comportamentais adaptativos É possível ser feliz ao longo do processo de envelhecimento, além das perdas, também se obtém ganhos, mais sabedoria, mais descontração. Num outro estudo realizado os autores identificaram que parte da satisfação dos idosos advém da participação em atividades religiosas, dos contatos sociais e do estabelecimento de vínculos positivos que essas atividades proporcionam.

0 sentimento de felicidade, tem sido objeto de estudo por parte da Psicologia positiva, sendo considerada uma emoção positiva. Um aspeto apontado pela Psicologia Positiva é a relação das instâncias de tempo passado, presente e futuro com a satisfação com a vida, segundo a qual as emoções positivas vinculadas ao futuro associam-se a sentimentos de otimismo, esperança, fé e confiança. Conclui 0 autor atrás referenciado, que numa perspetiva neurobiológica, a presença da felicidade ou satisfação, além de promover melhor relacionamento entre as pessoas, é capaz de combater os prejuízos que o stress causa na saúde, fortalecendo o sistema imunológico.

Os conceitos de felicidade e satisfação com a vida, embora muito interligados, diferem em aspetos relacionados com a cognição: a satisfação com a vida enfatiza o processo de avaliação, enquanto o sentimento de felicidade, mesmo com algum componente cognitivo, desenvolve-se com maior relevância a partir das emoções. 
Apesar desta particularidade, existe uma significativa inter-relação entre os conceitos de bem-estar subjetivo, felicidade e satisfação com a vida.

As evidências científicas no campo da saúde, através de estudos Iongitudinais realizados pelo Institute of Medicine (2011), têm demostrado a importância da felicidade e do otimismo no aumento da Iongevidade, na proteção de morbilidades e incapacidades e na tendência à adoção de comportamentos saudáveis. Evidenciam também que 0 sentimento de felicidade está fortemente associado à autoestima, com o conforto domiciliar, com 0 suporte e a participação social, sendo influenciado também pela situação conjugal.

As evidências consultadas, apontam inúmeros fatores alocados à conceção de bem-estar na velhice, sendo eles de ordem biológica, sociológica e psicológica, tais como: a longevidade, a saúde biológica e mental, a satisfação e a competência social, a continuidade nos papéis familiares e ocupacionais, as relações informais em grupos primários, entre outros elementos. Pesquisas sobre o ajustamento pessoal e social na velhice, concluem que envelhecer bem implicava em estar satisfeito com a sua vida atual e possuir expetativas positivas em relação ao futuro.

Podemos enquadrar as pessoas mais velhas no oitavo estádio proposto por Erik Erikson, na sua Teoria Psicossocial sobre o Desenvolvimento, designado por Integridade versus Desespero. Este estádio do desenvolvimento, é definido como sendo o último do ciclo vital, onde é feita uma reflexão e avaliação sobre o decurso da vida e a satisfação com a mesma. Obtendo-se duas possibilidades de desfecho, uma que dá primazia a um desfecho positivo, caraterizada pela satisfação e sentimento de felicidade e a outra possibilidade de desfecho negativo, onde predominam emoções negativas e marcada insatisfação com a vida.

Podemos consultar um número considerável de estudos relacionados aos aspetos negativos do envelhecimento, nomeadamente às perdas e doenças, em contraste com um baixo número de estudos sobre os ganhos associados ao processo de envelhecer, sendo que, as evidências revelam que a satisfação com a vida e a felicidade se constituem como variáveis essenciais na preservação e promoção do processo de envelhecimento gratificante.

Com a emergência da Gerontologia, passou a investigar-se também os aspetos positivos do envelhecimento saudável e gratificante e o potencial para o desenvolvimento de estratégias adaptativas bem sucedidas. Parecenos, assim, urgente olharmos para os mais velhos, como sujeitos capazes de vivenciarem sentimentos de autorrealização e felicidade.

\section{OBJETIVOS DE INVESTIGAÇÃO}

0 objetivo geral desta investigação assenta em analisar o nível de satisfação e felicidade dos idosos institucionalizados. Neste sentido, formularam-se como objetivos específicos os seguintes:

- Avaliar a satisfação com os cuidados dos idosos institucionalizados num concelho do Norte do país.

- Avaliar a felicidade dos idosos institucionalizados num concelho do Norte do país.

Estabeleceram-se, ainda, como hipóteses de investigação:

1. HI $\mathrm{H}_{1}$ : A satisfação com os cuidados dos idosos Institucionalizados é diferente segundo as variáveis de Contexto Sociodemográfico;

2. $\mathrm{H}_{2}$ : A satisfação com os cuidados dos idosos Institucionalizados é diferente segundo as variáveis de Contexto Situacional;

3. HI 3 : A satisfação com os cuidados dos idosos o é diferente segundo as variáveis de Contexto Clínico;

4. $\mathrm{H}_{4}$ : A felicidade dos idosos Institucionalizados é diferente segundo as variáveis de Contexto Sociodemográfico;

5. HI 5 : A felicidade dos idosos Institucionalizados é diferente segundo as variáveis de Contexto Situacional;

6. HI 6 : A felicidade dos idosos Institucionalizados é diferente segundo as variáveis de Contexto Clínico;

7. HI : Existe uma relação positiva direta entre a variável satisfação com os cuidados e a variável felicidade 


\section{SATISFAÇÃO COM OS CUIDADOS E FELICIDADE EM IDOSOS INSTITUCIONALIZADOS}

\section{AMOSTRA}

A população é constituída por 146 idosos e a amostra final por 61 (42\% da população), conforme apresentado na Tabela 1. Os 85 idosos que fazem parte da população e não estão na amostra foram excluídos por apresentarem perturbações mentais, sendo que essa informação foi dada pelas próprias instituiç̧̃̃es.

Tabela 1 - Distribuição da Amostra por Variáveis Sociodemográficas e Situacionais

\begin{tabular}{|c|c|c|c|}
\hline Variável & & $\mathrm{n}$ & $\%$ \\
\hline \multirow[t]{2}{*}{ Sexo } & Masculino & 15 & 24.6 \\
\hline & Feminino & 46 & 75.4 \\
\hline \multirow[t]{6}{*}{ Idade } & 65 a 69 anos & 6 & 9.8 \\
\hline & 70 a 74 anos & 3 & 4.9 \\
\hline & 75 a 79 anos & 13 & 21.3 \\
\hline & 80 a 84 anos & 17 & 27.9 \\
\hline & 85 a 89 anos & 18 & 29.5 \\
\hline & 90 ou mais anos & 4 & 6.6 \\
\hline \multirow[t]{3}{*}{ Estado civil } & Solteiro & 10 & 16.4 \\
\hline & Casado & 11 & 18.0 \\
\hline & Viúvo & 40 & 65.6 \\
\hline \multirow{3}{*}{$\begin{array}{l}\text { Grau de } \\
\text { escolaridade }\end{array}$} & Não foi à escola & 33 & 54.1 \\
\hline & 1. ${ }^{\circ}$ Ciclo & 25 & 41.0 \\
\hline & 2. ${ }^{\circ}$ Ciclo & 3 & 4.9 \\
\hline \multirow{2}{*}{$\begin{array}{l}\text { Satisfeito com o } \\
\text { valor de reforma }\end{array}$} & Sim & 35 & 57.4 \\
\hline & Não & 26 & 42.6 \\
\hline \multirow{6}{*}{$\begin{array}{l}\text { Tempo de } \\
\text { internamento }\end{array}$} & 1 a 12 meses & 17 & 27.9 \\
\hline & 13 a 24 meses & 11 & 18.0 \\
\hline & 25 a 36 meses & 8 & 13.1 \\
\hline & 37 a 48 meses & 7 & 11.5 \\
\hline & 48 a 60 meses & 3 & 4.9 \\
\hline & Mais de 60 meses & 15 & 24.6 \\
\hline \multirow{3}{*}{$\begin{array}{l}\text { Iniciativa da } \\
\text { institucionalização }\end{array}$} & Própria & 25 & 41.0 \\
\hline & Familiares & 27 & 44.3 \\
\hline & Técnicos de Ação Social & 9 & 14.8 \\
\hline \multirow{3}{*}{$\begin{array}{l}\text { Motivo da } \\
\text { institucionalização }\end{array}$} & Questões clínicas & 26 & 42.6 \\
\hline & Questões familiares e/ou pessoais & 34 & 55.7 \\
\hline & Outro & 1 & 1.6 \\
\hline \multirow{4}{*}{$\begin{array}{l}\text { Perceção do } \\
\text { convívio social }\end{array}$} & Muito satisfeito & 26 & 42.6 \\
\hline & Satisfeito & 30 & 49.2 \\
\hline & Insatisfeito & 4 & 6.6 \\
\hline & Muito insatisfeito & 1 & 1.6 \\
\hline
\end{tabular}

Verifica-se, pelos dados apresentados na Tabela 1, que a maioria dos respondentes é do sexo feminino, viúvo(a), não tem escolaridade, está satisfeito com a reforma que recebe e está satisfeito ou muito satisfeito com a instituição onde está institucionalizado. 


\section{METODOLOGIA E INSTRUMENTO DE RECOLHA DE DADOS}

Realizámos um estudo de caráter exploratório, descritivo e inferencial, utilizando como instrumento de recoIha de dados um questionário composto por três partes:

- A primeira parte foi um questionário sociodemográfico e clínico, com 16 questões.

- A segunda parte composta pelo questionário de avaliação da satisfação em estruturas residenciais para pessoas idosas (Instituto de Segurança Social, 2011), um questionário de autorrelato de 36 questões onde os itens 1 a 34 são respondidos numa escala de Likert de cinco pontos, variável entre 0 "Muito Insatisfeito" e 0 "Muito Satisfeito" e ainda a possibilidade de assinalar "Não Sei" (NS) ou "Não se Aplica" (NA). Este questionário avalia cinco dimensões: Fatores tangíveis; Fiabilidade; Capacidade de resposta; Confiança e segurança; e, Empatia. Os itens 35 e 36 são respondidos com "Sim" ou "Não".

- A terceira parte foi composta pelo Questionário da Felicidade de Oxford (Hills \& Argyle, 2002), um questionário de autorrelato de 29 itens, respondidos numa escala Likert de seis pontos sendo 10 menor grau de felicidade possível e 6 o maior.

Efetuaram-se testes de Shapiro-Wilk e observação visual da dispersão dos dados para definir as técnicas estatísticas a utilizar na análise inferencial, de acordo com as mais recentes práticas (Ghasemi \& Zahediasl, 2012; D. S. Moore et al., 2009; Thode, 2002). De acordo com os resultados obtidos, aplicaram-se as técnicas estatísticas apresentadas na Tabela 2.

Tabela 2 - Técnicas Estatísticas Aplicadas

\begin{tabular}{|c|c|}
\hline Objetivo ou Hipótese de Investigação & Técnica Estatística \\
\hline $\begin{array}{l}\mathrm{O}_{1} \text { Avaliar a Satisfação com os cuidados dos idosos } \\
\text { institucionalizados do concelho de Santa Marta de Penaguião }\end{array}$ & $\begin{array}{l}\text { Tabelas de } \\
\text { Frequências }\end{array}$ \\
\hline $\begin{array}{l}\mathrm{O}_{2} \text { Avaliar a Felicidade dos idosos institucionalizados do } \\
\text { concelho de Santa Marta de Penaguião }\end{array}$ & $\begin{array}{l}\text { Tabelas de } \\
\text { Frequências }\end{array}$ \\
\hline $\begin{array}{l}\mathrm{HI}_{1} \text { A Satisfação dos idosos Institucionalizados em Lares do } \\
\text { Concelho de Santa Marta de Penaguião é diferente segundo } \\
\text { as variáveis de Contexto Sociodemográfico }\end{array}$ & $\begin{array}{l}\text {-Student, Mann- } \\
\text { Whitney e Kruskal- } \\
\text { Wallis }\end{array}$ \\
\hline $\begin{array}{l}\mathrm{HI}_{2} \text { A Satisfação dos idosos Institucionalizados em Lares do } \\
\text { Concelho de Santa Marta de Penaguião é diferente segundo } \\
\text { as variáveis de Contexto Situacional }\end{array}$ & Kruskal-Wallis \\
\hline $\begin{array}{l}\mathrm{HI}_{3} \text { A Satisfação dos idosos Institucionalizados em Lares do } \\
\text { Concelho de Santa Marta de Penaguião é diferente segundo } \\
\text { as variáveis de Contexto Clínico }\end{array}$ & Kruskal-Wallis \\
\hline $\begin{array}{l}\mathrm{HI}_{4} \text { A Felicidade dos idosos Institucionalizados em Lares do } \\
\text { Concelho de Santa Marta de Penaguião é diferente segundo } \\
\text { as variáveis de Contexto Sociodemográfico }\end{array}$ & $\begin{array}{l}\text { Tabelas de } \\
\text { Frequências }\end{array}$ \\
\hline $\begin{array}{l}\mathrm{HI}_{5} \text { A Felicidade dos idosos Institucionalizados em Lares do } \\
\text { Concelho de Santa Marta de Penaguião é diferente segundo } \\
\text { as variáveis de Contexto Situacional }\end{array}$ & $\begin{array}{l}t \text {-Student e Kruskal- } \\
\text { Wallis }\end{array}$ \\
\hline $\begin{array}{l}\mathrm{HI}_{6} \text { A Felicidade dos idosos Institucionalizados em Lares do } \\
\text { Concelho de Santa Marta de Penaguião é diferente segundo } \\
\text { as variáveis de Contexto Clínica }\end{array}$ & Kruskal-Wallis \\
\hline $\begin{array}{l}\mathrm{HI}_{7} \text { Existe relação entre a variável Satisfação e a variável } \\
\text { Felicidade }\end{array}$ & $\begin{array}{l}\text { Correlações não } \\
\text { paramétricas de } \\
\text { Spearmann }\end{array}$ \\
\hline
\end{tabular}




\section{SATISFAÇÃO COM OS CUIDADOS E FELICIDADE EM IDOSOS INSTITUCIONALIZADOS}

Nos Procedimentos éticos, foi formalizado o pedido de autorização aos Presidentes de Direção das Instituições do concelho, reunindo assim o consentimento de todas as instituições para a realização da recolha de dados. Foram igualmente assegurados os pressupostos plasmados na Declaração de Helsínquia.

\section{RESULTADOS}

Para responder aos objetivos específicos conforme formulados calcularam-se as pontuações mínimas, máximas, medianas, médias e desvios padrão para as várias dimensões em estudo. Assim, para ir ao encontro do primeiro objetivo específico: "Avaliar a Satisfação com os cuidados dos idosos institucionalizados do concelho de Santa Marta de Penaguião", calcularam-se os valores apresentados na Tabela 3.

Tabela 3 - Valores mínimos, máximos, medianas, médias e desvios padrão para as cinco dimensões do questionário de avaliação da satisfação em estruturas residenciais para pessoas idosas

\begin{tabular}{llllc}
\hline & Mínimo & Máximo & Mediana & $\overline{\boldsymbol{x}} \pm \boldsymbol{s}$ \\
\hline Fatores tangíveis & 50.00 & 100 & 98.21 & $94.37 \pm 11.62$ \\
$\begin{array}{l}\text { Fiabilidade } \\
\begin{array}{l}\text { Capacidade de } \\
\text { resposta }\end{array}\end{array}$ & 16.67 & 100 & 75.00 & $72.26 \pm 21.53$ \\
$\begin{array}{l}\text { Confiança e } \\
\text { segurança }\end{array}$ & 50.00 & 100 & 84.38 & $81.90 \pm 11.80$ \\
\begin{tabular}{l} 
Empatia \\
\hline
\end{tabular} & 25.00 & 100 & 96.43 & $91.72 \pm 13.05$ \\
& & 100 & 95.00 & $91.16 \pm 18.32$ \\
\hline
\end{tabular}

Pode-se observar pelos dados na Tabela 3 que, em geral, a satisfação dos utentes das instituições em estudo é bastante alta, denotando-se a pontuação mais elevada em relação aos Fatores Tangíveis e a mais baixa em relação à Fiabilidade.

Para responder ao segundo objetivos específico: "Avaliar a Felicidade dos idosos institucionalizados do concelho de Santa Marta de Penaguião", calcularam-se os mesmos indicadores, mas desta vez para a variável dependente Felicidade Global, apresentando-se os resultados na Tabela 4.

Tabela 4 - Valores mínimos, máximos, medianas, médias e desvios padrão para a Felicidade Global

\begin{tabular}{llllc}
\hline \hline & Mínimo & Máximo & Mediana & $\overline{\boldsymbol{x}} \pm \boldsymbol{s}$ \\
\hline Felicidade Global & 2 & 6 & 4.10 & $4.07 \pm 0.72$ \\
\hline
\end{tabular}

Observando os dados apresentados na Tabela 4, conclui-se que em média os respondentes apresentam um nível de felicidade acima da média teórica (3.50), no entanto ainda muito abaixo daquilo que os autores da escala e vários outros autores consideram como o nível ideal de felicidade que se situa ligeiramente abaixo dos 6 (um 6 como pontuação média é considerado como "felicidade falsa" ou "demasiado feliz").

Para ir ao encontro das várias hipóteses de investigação, aplicaram-se as técnicas estatísticas, conforme apresentadas na Tabela 2. Assim, para verificar a primeira hipótese de investigação: "A Satisfação dos idosos Institucionalizados em Lares do Concelho de Santa Marta de Penaguião é diferente segundo as variáveis de Contexto Sociodemográfico", calcularam-se os valores de prova, apresentando-se os resultados na Tabela 5. 
Tabela 5 - Valores de prova para as cinco dimensões do questionário de avaliação da satisfação em estruturas residenciais para pessoas idosas, de acordo com as variáveis sociodemográficas

\begin{tabular}{lccccc}
\hline \hline & Sexo & Idade & Localidade & $\begin{array}{l}\text { Estado } \\
\text { civil }\end{array}$ & Escolaridade \\
\hline Fatores tangíveis & .219 & .337 & .157 & .400 & .384 \\
Fiabilidade & .513 & .487 & .373 & .836 & .611 \\
$\begin{array}{l}\text { Capacidade de } \\
\text { resposta }\end{array}$ & .328 & .821 & .978 & .245 & .796 \\
$\begin{array}{l}\text { Confiança e } \\
\text { segurança }\end{array}$ & .665 & .388 & .029 & .580 & .430 \\
Empatia & .512 & .734 & .023 & .699 & .459 \\
\hline
\end{tabular}

De acordo com os dados apresentados, existem diferenças estatisticamente significativas $(p<.05)$ somente para as dimensões Confiança e segurança e Empatia, entre respondentes com origem em diferentes localidades, sendo que o respondentes que viviam anteriormente em vilas ou cidades, pontuam inferior a quem vivia em meios mais pequenos (aldeia).

Para responder à segunda hipótese de investigação: "A Satisfação dos idosos Institucionalizados em Lares do Concelho de Santa Marta de Penaguião é diferente segundo as variáveis de Contexto Situacional", calcularam-se os valores prova apresentados na Tabela 6, verificando-se que não existem diferenças estatisticamente significativas $(p<.05)$ para nenhuma dimensão entre os respondentes de diferentes contextos situacionais.

Tabela 6 - Valores de prova para as cinco dimensões do questionário de avaliação da satisfação em estruturas residenciais para pessoas idosas, de acordo com as variáveis de contexto situacional

\begin{tabular}{llll}
\hline & $\begin{array}{l}\text { Tempo de } \\
\text { internamento }\end{array}$ & Iniciativa & Motivo \\
\hline Fatores tangíveis & .113 & .949 & .660 \\
Fiabilidade & .127 & .807 & .496 \\
Capacidade de resposta & .065 & .558 & .358 \\
Confiança e segurança & .218 & .935 & .591 \\
Empatia & .369 & .997 & .659 \\
\hline
\end{tabular}

Os valores prova para responder à terceira hipótese de investigação: "A Satisfação dos idosos Institucionalizados em Lares do Concelho de Santa Marta de Penaguião é diferente segundo as variáveis de Contexto Clínico", encontram-se na Tabela 7. 


\section{SATISFAÇÃO COM OS CUIDADOS E FELICIDADE EM IDOSOS INSTITUCIONALIZADOS}

Tabela 7 - Valores de prova para as cinco dimensões do questionário de avaliação da satisfação em estruturas residenciais para pessoas idosas, de acordo com as variáveis de contexto clínico

\begin{tabular}{lll}
\hline & Perceção da saúde & Atividade diária \\
\hline Fatores tangíveis & .245 & .001 \\
Fiabilidade & .070 & .867 \\
Capacidade de resposta & .083 & .480 \\
Confiança e segurança & .101 & .039 \\
Empatia & .036 & .217 \\
\hline
\end{tabular}

Observa-se, desta forma, que existem diferenças estatisticamente significativas $(p<.05)$ para as dimensões Fatores tangíveis e Confiança e segurança, relacionadas com a Atividade diária ( $p=.001$ e $p=.039$ respetivamente), em que os respondentes pouco ativos pontuam, em média, inferior aos restantes respondentes em ambas as dimensões e para a dimensão Empatia, relacionada com a Perceção de saúde em que os respondentes que têm uma perceção de saúde má pontuam em média inferior aos que têm uma perceção de saúde boa $(p=.036)$.

A quarta hipótese de investigação: "A Felicidade dos idosos Institucionalizados em Lares do Concelho de Santa Marta de Penaguião é diferente segundo as variáveis de Contexto Sociodemográfico", sendo os valores prova, de acordo com as técnicas estatísticas aplicadas, apresentadas na Tabela 8, verificando-se não existirem diferenças estatisticamente significativas para a dimensão Felicidade, de acordo com as variáveis sociodemográficas.

Tabela 8 - Valores de prova para a felicidade global, de acordo com as variáveis sociodemográficas

\begin{tabular}{lccccc}
\hline \hline & Sexo & Idade & Localidade & $\begin{array}{l}\text { Estado } \\
\text { civil }\end{array}$ & Escolaridade \\
\hline Felicidade & .434 & .067 & .122 & .904 & .575 \\
\hline
\end{tabular}

Para responder à quinta hipótese de investigação: "A Felicidade dos idosos Institucionalizados em Lares do Concelho de Santa Marta de Penaguião é diferente segundo as variáveis de Contexto Situacional", obtiveram-se os valores prova apresentados na Tabela 9, não se verificando diferenças estatisticamente significativas para nenhuma variável do contexto situacional.

Tabela 9 - Valores de prova para a felicidade global, de acordo com as variáveis de contexto situacional

\begin{tabular}{llll}
\hline \hline & $\begin{array}{l}\text { Tempo de } \\
\text { internamento }\end{array}$ & Iniciativa & Motivo \\
\hline Felicidade & .667 & .546 & .208 \\
\hline
\end{tabular}

Para a sexta hipótese de investigação: "A Felicidade dos idosos Institucionalizados em Lares do Concelho de Santa Marta de Penaguião é diferente segundo as variáveis de Contexto Clínica", cujos valores de prova, de acordo com o contexto clínico, são apresentados na Tabela 10, verificando-se uma diferença estatisticamente significativa entre respondentes de acordo com o nível da sua atividade diária $(p=.015)$.

Tabela 10 - Valores de prova para a felicidade global, de acordo com as variáveis de contexto clínico

\begin{tabular}{lll}
\hline & Perceção da saúde & Atividade diária \\
\hline Felicidade & .513 & .015 \\
\hline
\end{tabular}


No caso em apreço, são os respondentes com uma atividade diária muito ativa que apresentam uma felicidade superior aos restantes grupos.

Para a última hipótese de investigação: "Existe relação entre a variável Satisfação e a variável Felicidade", foram calculadas os índices de correlação de Spearmann, entre as cinco dimensões da Satisfação e a dimensão Felicidade, sendo os resultados apresentados na Tabela 11.

Tabela 11 - Índices de Correlação e Valores de Prova entre as cinco dimensões da Satisfação e a dimensão Felicidade

\begin{tabular}{|c|c|c|}
\hline \multirow{2}{*}{ Correlação de Spearmann } & \multicolumn{2}{|l|}{ Felicidade } \\
\hline & $\rho$ & Valor de prova \\
\hline Fatores tangíveis & .215 & .103 \\
\hline Fiabilidade & .108 & .535 \\
\hline Capacidade de resposta & .187 & .231 \\
\hline Confiança e segurança & .514 & $<.001$ \\
\hline Empatia & .320 & .037 \\
\hline
\end{tabular}

Verifica-se, pelos dados apresentados na Tabela 11, que existem correlações positivas e estatisticamente significativas entre as dimensões Confiança e segurança $(p<.001)$ e Empatia $(p=.037)$ e a dimensão Felicidade, sendo que estas têm uma magnitude moderada na combinação Confiança e segurança / Felicidade $(r=.514) \mathrm{e}$ fraca (Schober \& Schwarte, 2018) na combinação Empatia / Felicidade $(r=.320)$.

\section{DISCUSSÃO}

0 presente estudo mostra-nos que os respondentes das várias instituições abrangidas estão, no geral satisfeitos com a qualidade dos serviços que thes são prestados apresentando, no entanto, níveis de felicidade abaixo do desejável.

Contudo, 0 impacto que a institucionalização tem sobre a felicidade não é consensual, e a literatura tanto apresenta estudos em que idosos institucionalizados apresentam índices de felicidade superiores aos não institucionalizados com estudos que apresentam 0 contrário (Kok et al., 2015; K. Moore et al., 2007; Neocleous \& Apostolou, 2016; Rinnan et al., 2018).

Por outro lado, o presente estudo encontrou poucas diferenças estatisticamente significativas entre grupos, tanto para variáveis sociodemográficas, de contexto situacional e de contexto clínico, sendo certo que, não obstante tal invalidar a verificação de várias das hipóteses de investigação, tal não retira a importância dos resultados do mesmo (Amrhein et al., 2019). Aliás, o presente estudo lança, no nosso entender, um desafio para melhor estudar e entender o fenómeno da felicidade entre os idosos, institucionalizados ou não, propondo a realização de mais estudos, especialmente longitudinais, para avaliar o impacto de terapias ocupacionais específicas sobre os níveis de felicidade.

\section{CONCLUSÃO}

Dispomos desde 2017, da estratégia nacional para o envelhecimento ativo e saudável 2017-2025. Neste documento encontramos linhas orientadoras para a promoção do envelhecimento saudável e gratificante, no qual é recomendado que as pessoas percebam o seu potencial para o bem-estar físico, social e mental ao longo do curso da vida, e que essas pessoas participem na sociedade de acordo com suas necessidades, desejos e capacidades; ao mesmo tempo, propicia proteção, segurança e cuidados adequados, quando necessários.

Destaca o mesmo documento que, é necessário conhecer e avaliar a influência dos fatores determinantes durante o curso de vida, e consequentemente do processo de envelhecimento, de modo a aproveitar as transições e as "janelas de oportunidade" e estimular a saúde, a participação e segurança nos diferentes estádios do ciclo vital. Por exemplo, há evidências que demonstram que o estímulo e as relações afetivas seguras na infância 


\section{SATISFAÇÃO COM OS CUIDADOS E FELICIDADE EM IDOSOS INSTITUCIONALIZADOS}

influenciam a capacidade individual de aprendizagem e de convívio em sociedade durante todos os estádios posteriores da vida.

0 facto de 0 idoso estar institucionalizado em regime de lar de dia, facilita o vínculo com outros idosos, sendo este facto apontado como uma influência positiva na promoção do bem-estar subjetivo na autonomia e na liberdade do idoso.

0 envelhecimento saudável e gratificante prepara-se ao longo do ciclo vital, é possível viver o processo de envelhecimento de forma ativa e com satisfação e felicidade. Foi para nós muito gratificante obtermos resultados tão positivos neste grupo de pessoas mais velhas. Envelhecer não significa deixar de sentir momentos de felicidade, nem tão pouco deixar de percepcionar bem estar geral e satisfação com a vida. Ao longo do processo de envelhecer a pessoa continua o seu propósito de manter satisfação com a vida e consequentemente momentos de felicidade.

\section{REFERÊNCIAS BIBLIOGRÁFICAS}

Amrhein, V., Greenland, S., \& McShane, B. (2019). Retire statistical significance. Nature, 567(March), 7-9.

Baltes, P. B., \& Baltes, M. M. (2010). Psychological perspectives on successful aging: The model of selective optimization with compensation. Em P. B. Baltes \& M. Baltes (Eds.), Successful Aging (pp. 1-34). Cambridge University Press. https://doi.org/10.1017/cb09780511665684.003

Bronfenbrenner, U. (1977). Toward an Experimental Ecology of Human Development. American Psychologist, 32(7), 513-531. https://doi.org/10.1037/0003-066X.32.7.513

Bronfenbrenner, U. (1986). Ecology of the Family as a Context for Human Development. Developmental psychology, 22(6), 723-742.

Bronfenbrenner, U. (2005). Making human beings human: Bioecological perspectives on human development. Em The SAGE Program on Applied Developmental Science. Sage Publications.

Bronfenbrenner, U., \& Ceci, S. J. (1994). Nature-nuture reconceptualized in developmental perspective: A bioecological model. Psychological Review, 101(4), 568-586. https://doi.org/10.1037/0033-295X.101.4.568

Bronfenbrenner, U., \& Morris, P. A. (2007). The bioecological model of human development. Em Handbook of child psychology: Volume 1. Theoretical models of human development (pp. 793-828). John Wiley \& Sons, Inc. https://doi.org/10.1002/9780470147658.chpsy0114

Direção-Geral da Saúde. (2017). Estratégia Nacional para o Envelhecimento Ativo e Saudável - 2017-2025.

Ghasemi, A., \& Zahediasl, S. (2012). Normality tests for statistical analysis: A guide for non-statisticians. International Journal of Endocrinology and Metabolism, 10(2), 486-489.

Hills, P., \& Argyle, M. (2002). The Oxford Happiness Questionnaire: A compact scale for the measurement of psychological well-being. Personality and Individual Differences, 33(7), 1073-1082. https://doi.org/10.1016/S0191-8869(01)00213-6

Instituto de Segurança Social. (2011). Questionário de avaliação da satisfação de estrutura residencial para idoSOS.

Kok, L., Berden, C., \& Sadiraj, K. (2015). Costs and benefits of home care for the elderly versus residential care: a comparison using propensity scores. European Journal of Health Economics, 16(2), 119-131. https://doi.org/10.1007/s10198-013-0557-1

Moore, D. S., McCabe, G. P., \& Craig, B. A. (2009). Introduction to the Practice of Statistics. W. H. Freeman and Company.

Moore, K., Delaney, J. A., \& Dixon, M. R. (2007). Using Indices of Happiness To Examine the Influence of Environmental Enhancements for Nursing Home Residents With Alzheimer'S Disease. Journal of Applied Behavior Analysis, 40(3), 541-544. https://doi.org/10.1901/jaba.2007.40-541

Neocleous, G., \& Apostolou, M. (2016). Happiness in and out of nursing homes: The case of Cyprus. International Social Work, 59(4), 533-544. https://doi.org/10.1177/0020872815598567 
Neri, A. L. (2006). 0 legado de Paul B. Baltes à Psicologia do Desenvolvimento e do Envelhecimento. Temas em Psicologia, 14(1), 17-34.

Rinnan, E., André, B., Drageset, J., Garåsen, H., Espnes, G. A., \& Haugan, G. (2018). Joy of life in nursing homes: A qualitative study of what constitutes the essence of Joy of life in elderly individuals living in Norwegian nursing homes. Scandinavian Journal of Caring Sciences, 32(4), 1468-1476. https://doi.org/10.1111/scs.12598

Schober, P., \& Schwarte, L. A. (2018). Correlation coefficients: Appropriate use and interpretation. Anesthesia and Analgesia, 126(5), 1763-1768. https://doi.org/10.1213/ANE.0000000000002864

Thode, H. C. (2002). Testing for Normality. Marcel Dekker, Inc.

WHO. (2002). Active aeging: a policy framework. Em World Health Organization. OMS.

WHO. (2015). Envelhecimento ativo: uma política de saúde. Organização Pan-Americana da Saúde. 
